\title{
THERMAL PERFORMANCE OF THE SNS CRYOMODULE
}

\author{
E. F. Daly, J. P. Hogan, I. E. Campisi, M. Drury, D. Machie, J. Preble, \\ C. H. Rode, T. Whitlatch, K. M. Wilson, and M. Wiseman \\ Thomas Jefferson National Accelerator Facility (Jefferson Lab) \\ Newport News, VA, 23606, USA
}

\begin{abstract}
When complete, the Spallation Neutron Source (SNS) will provide a $1 \mathrm{GeV}, 2 \mathrm{MW}$ beam for experiments. One portion of the machine's linac consists of over 80 Superconducting Radio Frequency (SRF) $805 \mathrm{MHz}$ cavities housed in a total of 23 cryomodules $(\mathrm{CMs})$ operating at $2.1 \mathrm{~K}$. Minimization of the total heat load is critical to machine performance since the refrigerator capacity is fixed. The total heat load of the cryomodules consists of the fixed static load and the dynamic heat load which is proportional to the cavity performance. The heat load of the cryomodules is the single largest load to the cooling circuits of the refrigerator. During acceptance testing at Jefferson Lab, a series of measurements have been taken on the prototype and first three production cryomodules. Calorimetric measurements of the primary heat load and shield heat load are presented and discussed. Temperature measurements taken allow a comparison between actual and predicted thermal performance of two components unique to this cryomodule design: the helium gas-cooled fundamental power coupler (FPC) and the helium heat exchanger (HX).
\end{abstract}

\section{INTRODUCTION}

A six-laboratory consortium is providing components for the SNS at Oak Ridge National Laboratory [1]. The SNS linac employs SRF cavities to accelerate the particle beam from an energy of $185 \mathrm{MeV}$ up to $1 \mathrm{GeV}$ in a segment of tunnel approximately 200 meters in length. Jefferson Lab is providing twenty-three CMs, eleven $?=0.61$ and twelve $?=0.81 \mathrm{CMs}$ that contain three and four cavities respectively. The cavities must provide accelerating gradients between 10 and $16 \mathrm{MV} / \mathrm{m}$ during pulsed operation [2] while meeting the thermal performance requirements [3]. 




FIGURE 1. High Beta $(?=0.81)$ Cryomodule Flow Schematic

Thus far, one prototype and six production CMs have been built at Jefferson Lab. Acceptance tests to verify performance have been completed on the prototype, designated M0, and three production CMs, designated sequentially as M1, M2 and M3. The "M" prefix indicates a $?=0.61$ or medium beta $(\mathrm{MB})$ cryomodule. The prototype as well as two production CMs have been successfully shipped from Jefferson Lab and reside on-site at ORNL.

\section{CRYOMODULE HEAT LOADS}

\section{Flow Description}

The CM heat loads are absorbed by three helium streams flowing within the CM (FIGURE 1). The primary circuit ( 3 atm, $5 \mathrm{~K}$ supply) employs a heat exchanger to precool the fluid and a Joule-Thomson (J-T) valve to produce $2.1 \mathrm{~K}$ superfluid helium within the CM. There is a secondary circuit with $\mathrm{J}-\mathrm{T}$ valve control that provides coolant to the FPC outer conductors, and a third circuit (4 atm, $35 \mathrm{~K}$ supply) that provides coolant to the radiation shield. The secondary circuit, available for use because superfluid is produced in the $\mathrm{CM}$ rather than at the central helium liquifier (CHL), includes heat intercepts and a surge tank to minimize the potential for flow instabilities. The secondary valve is used to vary the flow through the coupler circuit for operations with and without RF power. After exiting the CM through the side of the vacuum vessel, the exhaust is routed back to the CHL via a warm helium gas return pipe. A metering valve, set on each exhaust stream, ensures that the cold flange on each coupler operates at the required temperature $(5-6 \mathrm{~K})$ and also balances the flow between the parallel coupler circuits. 


\section{Budgeted vs. Estimated Heat Loads}

For the primary circuit maintained at $2.1 \mathrm{~K}$, the heat loads are divided into three categories: 1) a static contribution consisting of solid conduction, residual gas conduction and radiation, 2) a dynamic contribution consisting of RF heating in the cavity and coupler and 3) a distribution system allotment that consists of u-tube and transfer line heat loads. There is no dynamic contribution to the $50 \mathrm{~K}$ shield and its cooling circuit. A comparison of the budgeted and estimated heat loads is provided in TABLE 1. For the MB CM, the total estimated heat load to the primary circuit is $72 \%$ of the budget per cryomodule, and for the shield circuit is $91 \%$ of the budget per cryomodule. The primary static heat load for the $\mathrm{MB} \mathrm{CM}$ is estimated as $9.7 \mathrm{~W}$ and includes contributions from bayonets, valves, supports, wiring, cabling and radiation. The dynamic heat load is estimated as $8.3 \mathrm{~W}$ and is divided evenly between the three cavities and FPCs housed within the cryostat.

\section{RESULTS AND DISCUSSION}

\section{K Static and RF Heat Loads}

The following measurement technique, similar to that employed during CEBAF cryomodule acceptance testing [4], is used to quantify the static heat load and RF heat load. With the primary J-T supply valve and u-tube return valve closed, the helium bath pressure rate-of-rise is measured during three conditions: with the helium bath heater on (HEATER), with only RF power on (RF) and with no heater or RF power (STATIC). Five separate 30-second measurements are made in the following order - STATIC, HEATER, STATIC, RF and then STATIC. Using a known heater power ( $\left.Q_{\text {HEATER }}\right)$ and the average rate-of-rise $(\mathrm{dP} / \mathrm{dt})$ for each condition, the static and $\mathrm{RF}$ heat loads are then calculated from the following equations:

$$
\begin{aligned}
& \mathrm{Q}_{\text {STATIC }}=\mathrm{Q}_{\text {HEATER }} *\left(\mathrm{dP} /\left.\mathrm{dt}\right|_{\text {STATIC }}\right) /\left(\mathrm{dP} /\left.\mathrm{dt}\right|_{\text {HEATER+STATIC }}-\mathrm{dP} /\left.\mathrm{dt}\right|_{\text {STATIC }}\right) \\
& \mathrm{Q}_{\mathrm{RF}}=\mathrm{Q}_{\text {HEATER }} *\left(\mathrm{dP} /\left.\mathrm{dt}\right|_{\text {RF+STATIC }}-\mathrm{dP} /\left.\mathrm{dt}\right|_{\text {STATIC }}\right) /\left(\mathrm{dP} /\left.\mathrm{dt}\right|_{\text {HEATER+STATIC }}-\mathrm{dP} /\left.\mathrm{dt}\right|_{\text {STATIC }}\right)(2) .
\end{aligned}
$$

Note that during this measurement, the flow to the secondary circuit for the FPC outer conductors ideally should be maintained constant. Actually the inlet temperature to the

\begin{tabular}{|c|c|c|c|c|c|c|c|c|}
\hline & \multicolumn{4}{|c|}{$\begin{aligned} ?= & 0.61 \text { Cryomodule } \\
& (3 \text { cavities })\end{aligned}$} & \multicolumn{4}{|c|}{$\begin{array}{c}?=\underset{ }{0.81 \text { Cryomodule }} \\
(4 \text { cavities })\end{array}$} \\
\hline & \multicolumn{2}{|c|}{$2.1 \mathrm{~K}$} & \multicolumn{2}{|c|}{$50 \mathrm{~K}$} & \multicolumn{2}{|c|}{$2.1 \mathrm{~K}$} & \multicolumn{2}{|c|}{$50 \mathrm{~K}$} \\
\hline Description & Budget & Estimate & Budget & Estimate & Budget & Estimate & Budget & Estimate \\
\hline Static & 15 & 9.7 & 146 & 131 & 28 & 11.5 & 146 & 161 \\
\hline $\begin{array}{l}\text { Dynamic } \\
\text { (RF On) }\end{array}$ & 14 & 8.3 & N/A & N/A & 20 & 17.1 & N/A & N/A \\
\hline $\begin{array}{l}\text { U-Tube \& } \\
\text { Distribution } \\
\text { Allotment }\end{array}$ & 10 & $<10$ & 24 & $<24$ & 10 & $<10$ & 30 & $<30$ \\
\hline Total & 39 & $<28$ & 170 & $<155$ & 58 & 38.6 & 200 & $<191$ \\
\hline
\end{tabular}

TABLE 1. Comparison of Heat Load Budget vs. Estimate for both SNS Cryomodules 


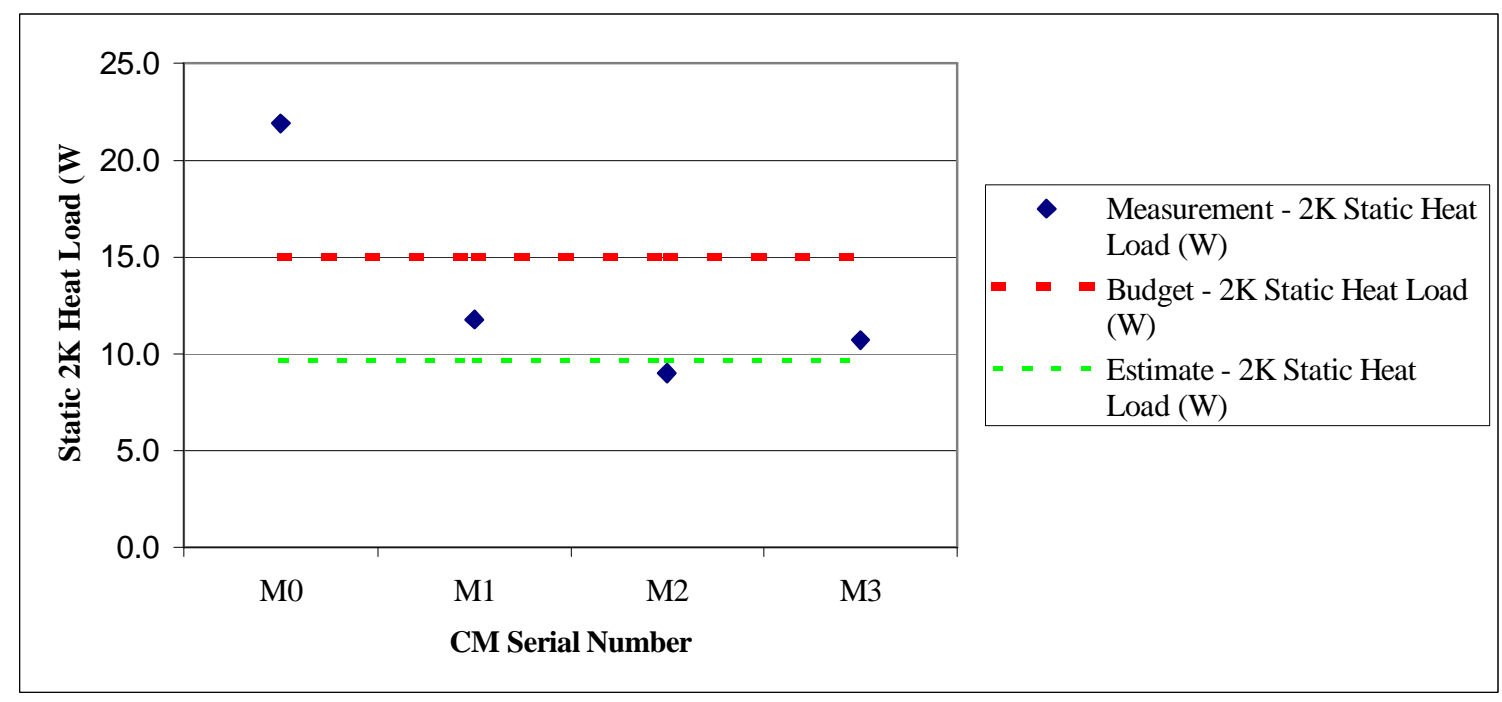

FIGURE 2. Measured Primary Static Heat Load vs. CM Serial Number

$\mathrm{CM}$ increases during the two and one half minute data acquisition cycle. Typically several measurements are taken before opening valves to recover the pressure. Over that time span the inlet temperature can increase by as much as $5-7 \mathrm{~K}$. There is a corresponding rise in the FPC cold flange temperature. This transient temperature more closely reflects the fluid temperature rather than the cold flange temperature since measurements have been made at relatively high gradients without indication of quenches.

The measurements are accurate to ? $1 / 2 \mathrm{~W}$ due in part to the accuracy of the capacitance manometer pressure transducer as well as the measured voltage and current on the cavity bath heater. Overall, the static heat load measurements agree well with the estimates; the average static load is approximately $10 \mathrm{~W}$ (FIGURE 2).

The measured RF heat load at the required gradient is approximately equivalent to the estimate for the prototype cavities, $2.7 \mathrm{~W}$, and is less than $1 \mathrm{~W}$ for each of the cavities in the three production CMs (FIGURE 3). One cavity, M3-3, was not measured due to schedule constraints.

The power dissipation scales quadratically with accelerating gradient. The required accelerating gradient is $15.5 \mathrm{MV} / \mathrm{m}$ for the $?=0.81$ cavities. Assuming no degradation due to field emission, the power dissipation will be more than two times greater for the higher

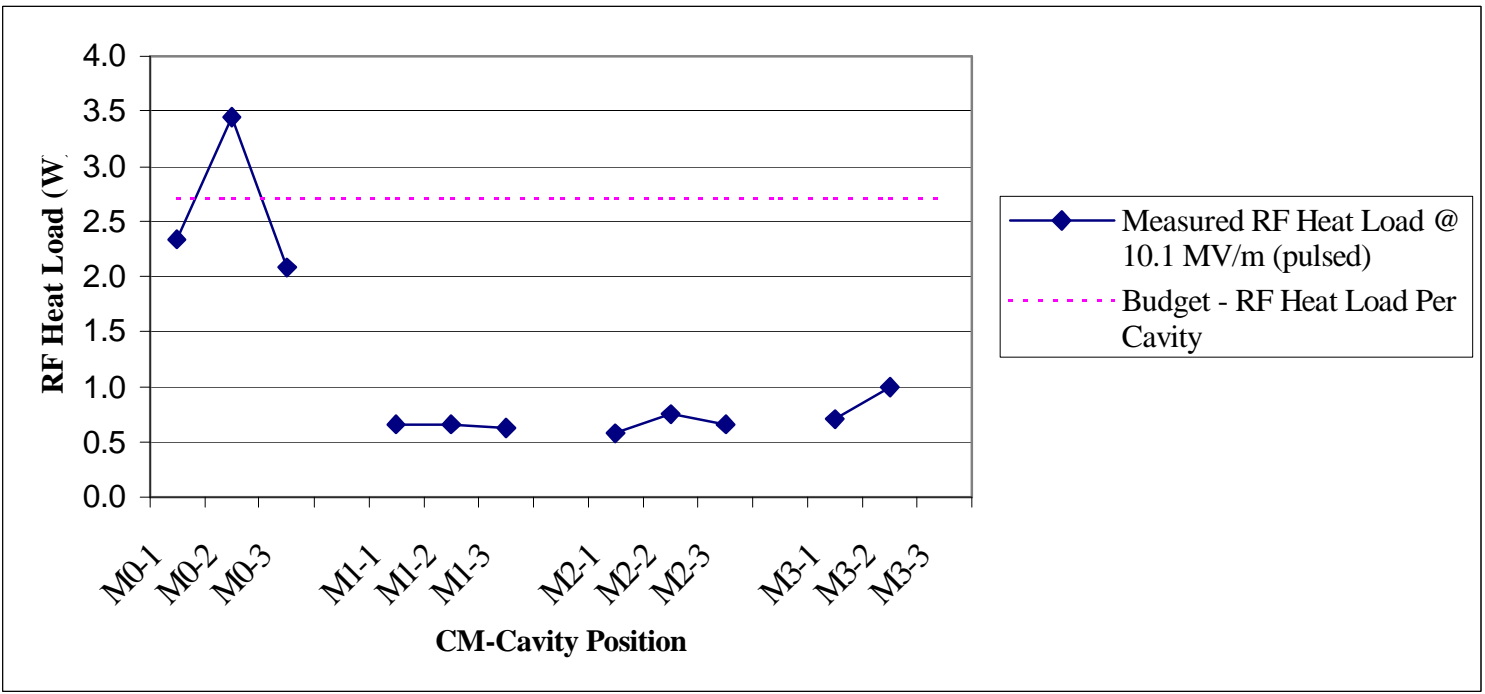

FIGURE 3. Measured RF Heat Load vs. CM-Cavity Position at $10.1 \mathrm{MV} / \mathrm{m}$ 


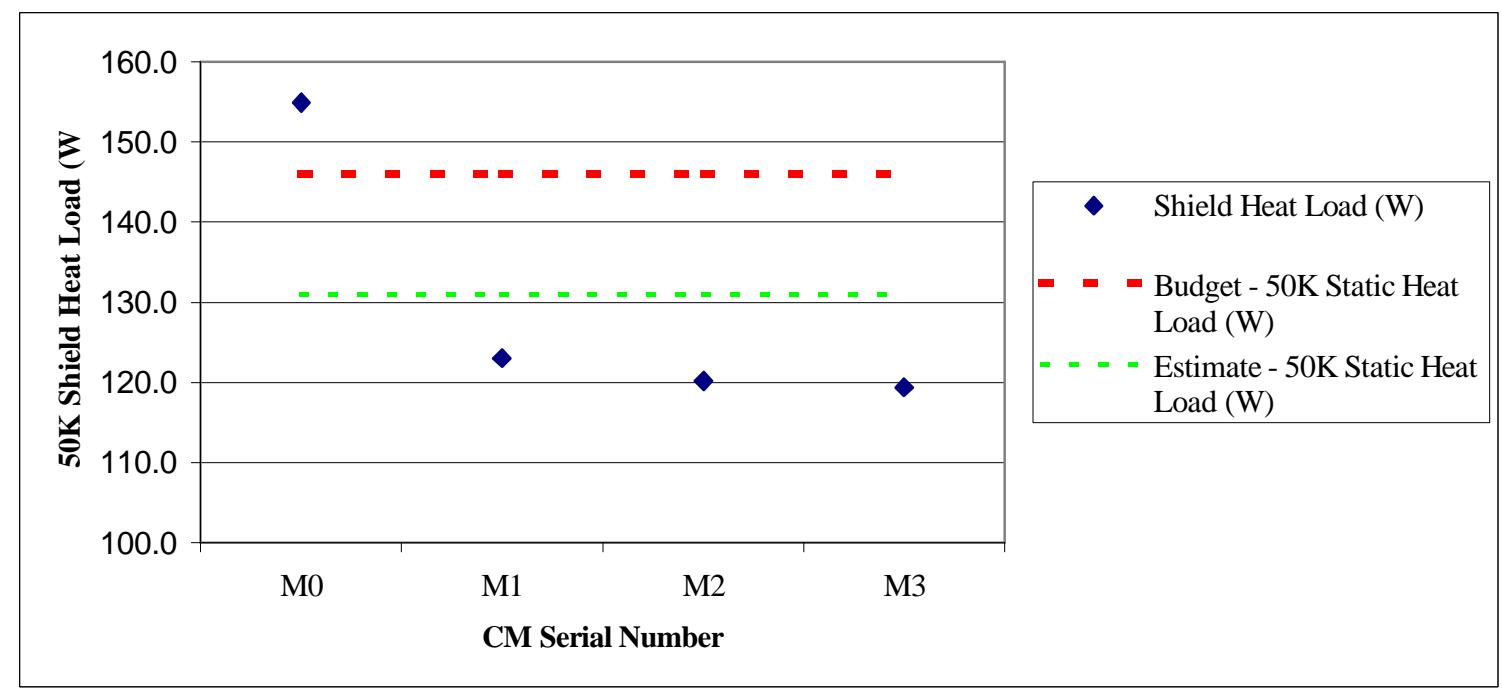

FIGURE 4. Measured Shield Heat Load vs. CM Serial Number

beta cavities. Initial vertical tests on these cavities have yielded acceptable results.

\section{K Shield Heat Load}

The measurements of the $50 \mathrm{~K}$ shield heat load are in good agreement with predictions (FIGURE 4). The measurement procedure included the following steps: first for a given valve position, monitor the gaseous helium inlet and outlet temperatures and the mass flow rate, then calculate the heat load using gaseous helium properties and time-averaged temperatures and flow rates. This procedure was repeated at several different flow rates. The accuracy of this type of measurement, approximately ? $10 \%$, is driven by the helium mass flow meter.

The heat load measured in the prototype CM (M0) is $129 \%$ higher compared with measurements on the production CMs. This is most likely caused by increased residual gas conduction in the insulating vacuum space due to the large helium leak rate that had been detected in the prototype shield process piping after cooldown. The prototype required active turbo-pumping during the entire test period to maintain the vacuum in the range of $10^{-6}$ torr. In addition, changes in the multi-layer insulation blanket design and improved blanket installation techniques contributed to the lower overall heat load in the production CMs.

\section{Fundamental Power Coupler Thermal Performance}

The primary requirement for the FPC is to transmit a maximum of $550 \mathrm{~kW}$ of RF power at a $7 \%$ duty cycle. Adding a $10 \%$ engineering margin, the design goal for the average power handling capability became $53 \mathrm{~kW}$. Another important requirement is to insert the coupler from the bottom to minimize particulate introduction and generation in the cavities. The outer conductor has counterflowing helium gas cooling to intercept the static and RF heat loads. The maximum flow rate is 0.075 grams per second per coupler. The flow passages are sized to keep the flow velocity high enough to overcome buoyancy forces and potential flow instabilities.

To verify the design and confirm the thermal calculations, the FPC installed on the M0-2 cavity was instrumented to measure the temperature profile along the outer conductor. In addition, the exhaust helium mass flow rate was measured during this 


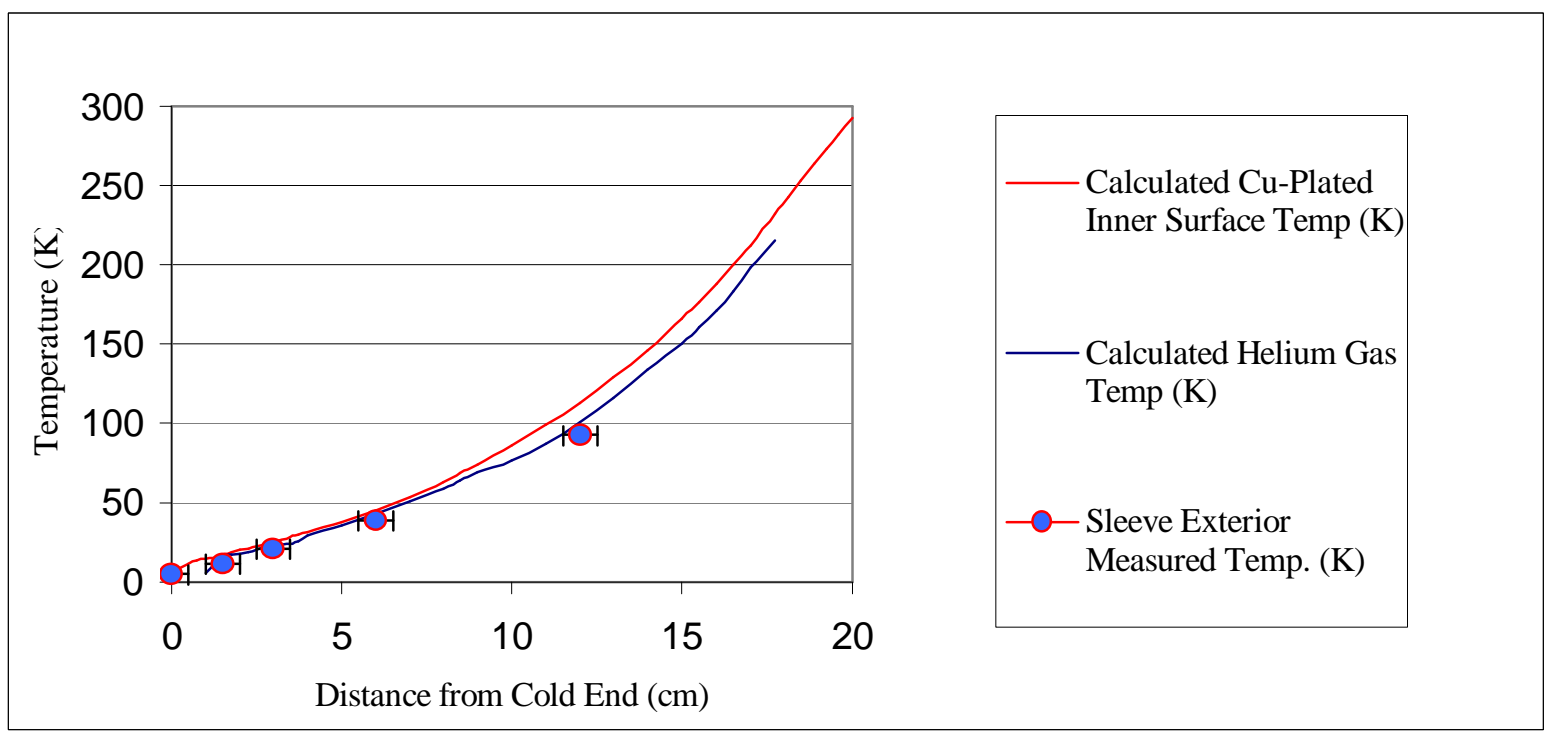

FIGURE 5. Comparison of Analytical and Measured Temperatures on the M0-2 FPC Outer Conductor

particular test to determine the maximum power handling capability of the FPC. With a stub tuner installed, the FPC transmitted $9 \mathrm{~kW}$ of $\mathrm{CW}$ power to the cavity. The resonant condition created by the stub tuner settings resulted in a transmitted power equivalent to $100 \mathrm{~kW}$ with the FPC, nearly double the design goal.

The thermal model used the cold and warm end temperatures, the helium mass flow rate and the equivalent transmitted RF power during the test as inputs. The results from the thermal model agree well with the experimental temperatures (FIGURE 5). The calculated conductive heat load transmitted to the cavity from the outer conductor during the higher power operation was estimated as $1.1 \mathrm{~W}$ - the expected conductive heat load at nominal operating conditions is less than $0.25 \mathrm{~W}$.

\section{Helium Heat Exchanger}

The HX is located in CM return end can to increase overall cryogenic system efficiency. This enables the SNS supply transfer lines to contain supercritical helium at $5 \mathrm{~K}$ as compared with $\sim 3 \mathrm{~K}$ for the CEBAF machine. The HX, a brazed-aluminum plate-fin construction, pre-cools the helium upstream of primary JT valve to increase liquid yield. The effectiveness is specified as greater than $90 \%$ and the assembly is required to be leak tight to $1 \times 10^{-9}$ mbar*liter/sec.

Initial Measurements indicate that performance is consistent with estimates. The measured inlet and outlet temperatures on the low-pressure-side of the HX are $2.0 \mathrm{~K}$ and $3.5 \mathrm{~K}$ respectively, compared with estimates of $2.1 \mathrm{~K}$ and $3.96 \mathrm{~K}$. Inlet and outlet temperatures for both streams are required to calculate the effectiveness of the counter-flow HX. The high-pressure-side inlet temperature indication is not reliable due to the placement of the silicon diode temperature sensor.

\section{CONCLUSIONS}

Measurements of thermal performance have been completed on the prototype and three production CMs. Results obtained for the static, RF and shield heat loads are in good agreement with estimates and are within budgeted heat loads. Measurements of the outer conductor thermal profile are in good agreement with calculations and indicate that the heat load from the FPC is acceptable. The FPC and its outer conductor can transmit 
approximately two times more than the required maximum RF power expected from the

SNS klystrons. Initial measurements of the available heat exchanger terminal temperatures agree with predictions. The heat exchanger performance assessment is incomplete; more study is required to quantify the heat exchanger effectiveness.

In addition, better $\mathrm{CM}$ and cavity construction techniques and procedures have incrementally improved the thermal performance.

\section{FUTURE WORK}

More testing is planned for the balance of the $\mathrm{MB} \mathrm{CMs}$, and current plans include testing only one HB CM from now through the conclusion of $\mathrm{CM}$ production.

\section{ACKNOWLEDGMENTS}

This work is supported by US DOE under Contract No. DE-AC05-00OR22725. Thanks to the Jefferson Lab Cryomodule Testing Team and the Cryomodule Production Group for their diligence and superior effort. Finally, special thanks to one particular coauthor, John Hogan, for presenting this paper at the conference on very short notice.

\section{REFERENCES}

1. T. E. Mason, “The Spallation Neutron Source: A Powerful Tool for Materials Research,” PAC2001, Chicago, IL, June 2001.

2. W. J. Schneider et al., "Design of the Spallation Neutron Source (SNS) Cryomodule," PAC2001, Chicago, IL, June 2001.

3. E. F. Daly et al., "Spallation Neutron Source Cryomodule Heat Loads and Thermal Design" in Advances in Cryogenic Engineering, Vol. 47, American Institute of Physics, New York (2002), p 531-539.

4. W. Schneider et al., "Thermal Performance of the CEBAF Superconducting Linac Cryomodule" in Advances in Cryogenic Engineering, Vol. 39, Plenum Press, New York (1994), p 589-597.

5. J. P. Hogan et al., "Thermal Optimization in SRF Cryomodule Production," Proceedings from this conference. 